\title{
Serological identification of HSP105 as a novel non-Hodgkin lymphoma therapeutic target
}

\author{
Roberta Zappasodi, ${ }^{1}$ Italia Bongarzone, ${ }^{2}$ Gaia C. Ghedini, ${ }^{3}$ Lorenzo Castagnoli, ${ }^{3}$ Antonello D. Cabras, ${ }^{4}$ Antonella Messina, ${ }^{5}$ \\ Monica Tortoreto, ${ }^{6}$ Claudio Tripodo, ${ }^{7}$ Michele Magni, ${ }^{1}$ Carmelo Carlo-Stella, ${ }^{1-8}$ Alessandro M. Gianni, ${ }^{1-8}{ }^{*}$ Serenella M. Pupa, ${ }^{3}$ \\ and ${ }^{*}$ Massimo Di Nicola ${ }^{1}$

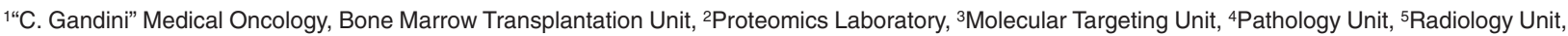 \\ ${ }^{6}$ Molecular Pharmacology Unit, Fondazione Istituto di Ricovero e Cura a Carattere Scientifico, Istituto Nazionale per lo Studio e la Cura dei Tumori, Milan, Italy; \\ ${ }^{7}$ Tumor Immunology Unit, Department of Health Science, Human Pathology Section, School of Medicine, University of Palermo, Palermo, Italy; and ${ }^{8}$ School of \\ Medical Oncology, University of Milan, Milan, Italy
}

We reported that the clinical efficacy of dendritic cell-based vaccination is strongly associated with immunologic responses in relapsed B-cell non-Hodgkin lymphoma (B-NHL) patients. We have now investigated whether postvaccination antibodies from responders recognize novel shared NHL-restricted antigens. Immunohistochemistry and flow cytometry showed that they cross-react with allogeneic BNHLs at significantly higher levels than their matched prevaccination samples or nonresponders' antibodies. Western blot analysis of DOHH-2 lymphoma proteome revealed a sharp band migrating at approximately 100 to $110 \mathrm{kDa}$ only with postvaccine repertoires from responders. Mass spectrometry identified heat shock protein-105 (HSP105) in that molecular weight interval. Flow cytometry and immunohistochemistry disclosed HSP105 on the cell membrane and in the cytoplasm of B-NHL cell lines and 97 diagnostic specimens. A direct correlation between HSP105 expression and lymphoma aggressiveness was also apparent. Treatment of aggressive human B-NHL cell lines with an anti-HSP105 antibody had no direct effects on cell cycle or apoptosis but significantly reduced the tumor burden in xenotransplanted immunodeficient mice. In vivo antilymphoma activity of HSP105 engagement was associated with a significant local increase of Granzyme $\mathrm{B}^{+}$killer cells that very likely contributed to the tumor-restricted necrosis. Our study adds HSP105 to the list of nononcogenes that can be exploited as antilymphoma targets. (Blood. 2011;118(16): 4421-4430)

\section{Introduction}

B-cell non-Hodgkin lymphomas (B-NHLs) are lymphoproliferative malignancies that encompass different biologic and histologic subtypes and arise from mature B cells within secondary lymphoid organs. ${ }^{1,2}$ Although these tumors are highly sensitive to both chemotherapy and radiotherapy, ${ }^{3}$ the ultimate goal of curing all NHLs remains to be attained. The last 20 years have demonstrated that the chimeric monoclonal antibody $(\mathrm{mAb})$ rituximab has been the most valuable addition to the B-NHL treatment armamentarium. At present, its combination with poly-chemotherapy still represents the standard of choice for the treatment of both indolent and aggressive B-NHL., ${ }^{4}$ However, given the difficulties in the management of relapse and resistance to rituximab ${ }^{6,7}$ and the late toxicities associated with its administration, ${ }^{8}$ it seems that treatment of B-NHL may have reached a new plateau. Alternative approaches as well as new molecular targets are thus required to ameliorate management and clinical outcome of the many patients that become resistant to rituximab.

We have recently reported that vaccination with autologous dendritic cells (DCs) pulsed with autologous killed tumor cells elicited clinical responses associated with tumor-specific immune activation in 6 of 18 relapsed indolent B-NHL patients. ${ }^{9}$ This points to the existence of novel, NHL-restricted therapeutic antigens. Immunohistochemistry (IHC) using patients' circulating immuno- globulins (Igs) on autologous lymphoma biopsies showed that our protocol induced tumor-restricted $\mathrm{Ab}$ responses only when it was clinically efficacious. ${ }^{9}$

In the present study, we have looked to see whether the antitumor humoral immunity developed in responders was directed against shared NHL-restricted antigen(s). Demonstration of such cross-reactivity constituted the prerequisite for exploitation of the responders' Ab repertoires to discover new lymphoma antigens to be targeted in NHL immunotherapy. We have used an appropriately modified serological proteome-based approach (SERPA) to identify the proteins differentially recognized by these repertoires. HSP105 was one of these proteins. It has been extensively shown that HSP105/110, together with HSP90, HSP70, HSP60, HSP40, and HSP27 classes of proteins, guide the normal folding, intracellular disposition, and proteolytic turnover of many of the key regulators of cell growth, differentiation, and survival, including HER2, mutant HER1, c-KIT, BCR-ABL, Akt, Cdk4, BRAF, HIF1- $\alpha$, p53, and other oncoproteins. ${ }^{10-12}$ Despite the focus on oncogenes as cancer therapy targets, sound experimental evidence demonstrates that targeting stress proteins could also be effective by promoting the destabilization of their client proteins. ${ }^{10,13-16}$

Our findings show that HSP105 is immunogenic in NHL oncotype and expressed on the plasma membrane and in the
Submitted June 29, 2011; accepted August 11, 2011. Prepublished online as Blood First Edition paper, August 22, 2011; DOI 10.1182/blood-2011-06-364570.

${ }^{\star}$ S.M.P. and M.D.N. contributed equally to this study.

The online version of this article contains a data supplement.
The publication costs of this article were defrayed in part by page charge payment. Therefore, and solely to indicate this fact, this article is hereby marked "advertisement" in accordance with 18 USC section 1734.

C 2011 by The American Society of Hematology 
cytoplasm of human B-NHL cell lines and specimens, with significantly higher intensity in highly proliferating aggressive lymphoma. In addition, its direct targeting by a specific Ab significantly impairs lymphoma growth and causes extensive necrosis of tumor and associated stroma cells, probably through the recruitment of Granzyme B-producing natural killer (NK) cells at the tumor site. Taken as a whole, our results point to HSP105 as a novel, attractive candidate biotarget for B-NHL therapy.

\section{Methods}

\section{Cohort samples}

The pre- and the postvaccination $\mathrm{Ab}$ repertoires from 7 of 18 patients with a history of relapsed indolent B-NHL enrolled in our previous pilot trial of DC-based vaccination (nos. 1, 2, 5, 8, 13, 14, and 17) $)^{9}$ were analyzed. Four were responders (2 complete and 2 partial remissions) according to the NHL International Workshop Criteria. ${ }^{17}$ Patients' serum was collected from peripheral blood $(\mathrm{PB})$, harvested before and 6 months after the last injection of the vaccine. Malignant B cells had been isolated from diagnostic tumor biopsies using a high-gradient immunomagnetic technique, according to the manufacturer's instructions (CD19 microbeads; Miltenyi Biotec), and had been cryopreserved until used. Written informed consent for the investigational use of lymphocytes, serum samples, and tumor tissue had been obtained in accordance with the Helsinki Declarations revised and amended in 2008 .

\section{Cell lines and culture conditions}

The following human neoplastic cell lines were used: DOHH-2 and SC-1 (follicular lymphoma [FL]); RL-19, SU-DHL-6, KARPAS-422, and SUDHL-4 (transformed diffuse large B-cell lymphoma [DLBCL]); GRANTA519 (mantle cell lymphoma); RAJI and NAMALWA (Burkitt lymphoma [BL]); HDMYZ and L-540 (Hodgkin lymphoma); KMS-11 (multiple myeloma), MOLT-4 (acute T lymphocytic leukemia), and SU-DHL-1 (anaplastic large $\mathrm{T}$ cell lymphoma). Cell lines were purchased from DSMZ and were routinely maintained in RPMI 1640 medium (Lonza Switzerland) supplemented with 10\% (volume/volume) FBS (Lonza Switzerland) and L-glutamine (Lonza Switzerland) in a humidified chamber with $5 \% \mathrm{CO}_{2}$ at $37^{\circ} \mathrm{C}$.

\section{Immunohistochemistry}

To evaluate NHL-specific cross-reactivity of patients' Ab repertoires, total Igs were purified from serum samples of 7 vaccinated patients and 8 healthy donors, conjugated with biotin and used for IHC as described. ${ }^{9}$

IHC evaluation of HSP105 and Ki-67 expression was performed on tissue sections of indolent (54 cases), aggressive B-NHL (43 cases), or nonmalignant lymph nodes ( 26 cases) obtained from the Tissue Bank of Fondazione Istituti di Ricovero e Cura a Carattere Scientifico (IRCCS) Istituto Nazionale dei Tumori di Milano. For these stainings, epitope retrieval was performed on formalin-fixed and paraffin-embedded tissue sections treated with $6 \mathrm{mM}$ citrate buffer for 6 minutes at $96^{\circ} \mathrm{C}$ or $2 \mathrm{mM}$ EDTA (pH 8) for 15 minutes at $98^{\circ} \mathrm{C}$, respectively. Endogenous peroxidase activity was blocked by incubation with $30 \%$ hydrogen peroxide in PBS for 40 minutes, followed by treatment with normal goat serum (1:50; Dako Denmark) for 40 minutes at room temperature. Mouse anti-human Ki-67 mAb (1:100; Dako Denmark) and rabbit polyclonal anti-human HSP105 Ab (1:1500, clone N-187; Santa Cruz Biotechnology) were, respectively, incubated for 1 hour at room temperature or overnight at $4^{\circ} \mathrm{C}$. Biotinconjugated anti-mouse or antirabbit $\operatorname{IgG}(1: 100$ and 1:200, respectively; Dako Denmark) was added for 1 hour at room temperature, and the immunoreactivity was revealed by HRP-conjugated streptavidin (1:300; Dako Denmark) and visualized using 3,3'-diaminobenzidine (SigmaAldrich). Tissue sections were lastly counterstained with hematoxylin. Anti-Ki-67 Ab immunoreactivity was scored as weak (5\%-10\%), moderate (10\%-30\%), and strong (>30\%) according to the number of positive nuclei in tumor cells. A combining score (CS) was arbitrarily defined by the pathologists to quantify HSP105 expression (0-2 indicates low; 3-5,

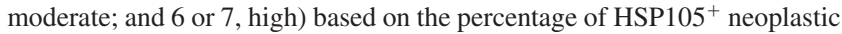
cells $(0$ indicates $\leq 1 \% ; 1,>1 \%$ and $\leq 20 \% ; 2,>20 \%$ and $\leq 50 \%$; $3,>50 \%$ and $\leq 80 \%$; and $4,>80 \%)$ and HSP105 staining intensity (1-3; 1 indicates weak; 2 , moderate; and 3 , strong).

\section{Flow cytometry}

Flow cytometry was performed on live primary malignant B cells isolated from tumor biopsies, live or fixed malignant B- and T-cell lines, and pooled donor PB mononuclear cells (PBMCs). Cells were incubated with $\mathrm{FcR}$ blocking reagent (Miltenyi Biotec) in the presence of biotin-conjugated human Igs $(100 \mu \mathrm{g} / \mathrm{mL})$ or rabbit polyclonal anti-human HSP105 Ab ( $4 \mu \mathrm{g} / \mathrm{mL}$; Santa Cruz Biotechnology) for 40 minutes on ice. FITC-labeled streptavidin (1:80; BD Biosciences PharMingen) and goat anti-rabbit IgG (KPL) were used to reveal the specific reactivity of primary Abs. Cytoplasmic stainings were carried out after fixation and permeabilization using BD Cytofix/Cytoperm (BD Biosciences PharMingen), according to the manufacturer's instructions. PBMCs were concurrently stained with peridinin chlorophyll protein-labeled CD3 and allophycocyanin-labeled CD19 (BD Biosciences) to detect HSP105 expression in CD3- or CD19gated subsets in multiparametric flow cytometry evaluations. To obtain absolute cell counts, cell samples were supplemented with a fixed amount of Flow-Count beads (Beckman Coulter) and stained with propidium iodide (PI). Cell counts per microliter were calculated from the following equation: counted viable cells $\times$ total beads/counted beads. Flow cytometry of apoptosis was performed as described. ${ }^{18}$ For cell-cycle analysis, cells were fixed in $70 \%$ ethanol and DNA was stained by overnight incubation with $2.5 \mu \mathrm{g} / \mathrm{mL}$ PI (Calbiochem) in the presence of $25 \mu \mathrm{g} / \mathrm{mL}$ RNAse (Sigma-Aldrich) at $4^{\circ} \mathrm{C}$. Data were acquired using BD CellQuest Version 3.3 software (BD Biosciences) and processed with the FlowJo Version 8.7.1 software (TreeStar) version for Macintosh Pro personal computer.

\section{OFFGEL fractionation}

DOHH-2 whole lysate $(1 \mathrm{mg})$ was isoelectrofocused using a 3100 OFFGEL Fractionator (Agilent Technologies) with an immobilized $\mathrm{pH} 3$ to 10 gradient strip (GE Healthcare) and a 24-well manifold, according to the manufacturer's instructions. ${ }^{19}$ Focusing was stopped after total voltage reached $64 \mathrm{kV} / \mathrm{h}$, and protein fractions were purified with 2-dimensional clean-up kit (GE Healthcare) and dissolved in Laemmli buffer. Nine-tenths of the volume was resolved by 1-dimensional SDS-PAGE, followed by silver staining, and one-tenth was transferred to a nitrocellulose membrane according to standard procedures.

\section{Western blot and immunoprecipitation}

Normal $\mathrm{CD}^{+} \mathrm{T}$ and $\mathrm{CD} 19^{+} \mathrm{B}$ cells, immunomagnetically selected from donor PB (Pan T-cell isolation kit and CD19 microbeads; Miltenyi Biotec), and tumor cell lines were solubilized for 40 minutes at $0^{\circ} \mathrm{C}$ with Laemmli buffer, as described. ${ }^{18}$

For immunoprecipitation, DOHH-2 lysates were precleared for $30 \mathrm{~min}-$ utes at $4{ }^{\circ} \mathrm{C}$ with immunopure immobilized protein A/G Sepharose (Pierce Chemical) previously equilibrated in lysis buffer. Protein extracts $(3 \mathrm{mg} /$ sample) were incubated for 2 hours at $4^{\circ} \mathrm{C}$ with $3 \mu \mathrm{g}$ rabbit polyclonal anti-HSP105 Ab (Santa Cruz Biotechnology) or rabbit normal serum as negative control, and then with protein A/G-Sepharose $(60 \mu \mathrm{L} / \mathrm{sample}$, previously equilibrated in lysis buffer) overnight at $4^{\circ} \mathrm{C}$ on a rocker. Sepharose-bound immunocomplexes were washed 3 times with lysis buffer and eluted in loading buffer.

Eluted immunoprecipitated proteins, OFFGEL protein fractions or lymphoma cell lysates, mixed with loading buffer and denatured on heating for 5 minutes at $95^{\circ} \mathrm{C}$, were subjected to electrophoresis on $4 \%-12 \%$ or $3 \%-8 \%$ NuPAGE Bis-Tris precast polyacrylamide gels (Invitrogen). Separated proteins were transferred onto nitrocellulose membrane (Hybond-C Super; GE Healthcare) in 20\% ethanol NuPAGE transfer buffer (Invitrogen), stained with Ponceau red solution, washed extensively with $0.5 \%$ Triton $\mathrm{X}-100 \mathrm{PBS}$, and saturated overnight at $4^{\circ} \mathrm{C}$ in blocking solution $(5 \%$ 
low-fat milk, $0.1 \%$ Triton X-100 PBS) before incubation with biotinconjugated patients' Igs (100 $\mu \mathrm{g} / \mathrm{mL})$, rabbit polyclonal antihuman HSP105 $\mathrm{Ab}(1 \mu \mathrm{g} / \mathrm{mL}$, Santa Cruz Biotechnology), mouse monoclonal antihuman HSP105 mAb (1:1000, Abcam), or rabbit polyclonal anti-human actin Ab (1:1000, Sigma-Aldrich) in blocking solution for 1 hour at room temperature. Immunoreactive proteins were visualized with appropriate secondary Abs: HRP-conjugated donkey anti-rabbit Igs (1:10 000; GE Healthcare) or HRP-conjugated streptavidin (1:10 000; GE Healthcare), and signals were detected using an enhanced ECL system (ECL Western Blotting Detection Reagents; GE Healthcare), according to the manufacturer's protocol.

\section{In-gel tryptic digestion, MALDI-TOF-MS}

To identify the proteins differentially recognized by postvaccination Igs of responders within the 1-dimensionally separated DOHH-2 fractions, protein bands were excised from silver-stained preparative gel and analyzed by matrix-assisted laser desorption/ionization time-of-flight mass spectrometry (MALDI-TOF-MS), as described. ${ }^{20}$ Briefly, proteins were in-gel digested with trypsin $(13 \mathrm{ng} / \mu \mathrm{L})$ for 18 hours at $37^{\circ} \mathrm{C}$. The peptide mixture obtained from each band was analyzed with MALDI-TOF Voyager-DE STR (Applied Biosystems), equipped with a nitrogen laser $(337 \mathrm{~nm})$. Spectra were analyzed with Aldente software (http://web.expasy.org/cgi-bin/ aldente/help.pl?intro.html). Input was searched according to the UniProtKB/ SwissProt database (predefined taxon: mammalia; spectrometer internal error max: 25). Only proteins identified in at least 3 independent experiments were considered.

\section{In vivo experiments}

Severe combined immunodeficiency (SCID) mice (6-8 weeks old) with body weight of 20-25 $\mathrm{g}$ were purchased from Charles River.

Mice were injected subcutaneously with $10 \times 10^{6}$ NAMALWA (54 mice) and SU-DHL-4 (36 mice) cells. When tumors reached 0.05 to $0.07 \mathrm{~cm}^{3}$, mice were randomized in 3 groups (6 mice/group, for a total of 3 and 2 experiments with NAMALWA and SU-DHL-4 models, respectively) to receive 3 intraperitoneal injections of $1 \mathrm{mg} / \mathrm{mL}$ rabbit polyclonal antihuman HSP105 or matched rabbit IgG isotype $(250 \mu \mathrm{g} /$ mouse, glyceroland azide-free preparation for in vivo use, Santa Cruz Biotechnology) or $\mathrm{NaCl}$ solution $0.9 \%$ ( $250 \mu \mathrm{L} /$ mouse). Tumors were calibrated twice a week, and tumor volume was calculated as $0.5 \times \mathrm{d} 1^{2} \times \mathrm{d} 2$, where $\mathrm{d} 1$ and $\mathrm{d} 2$ are the shortest and the longest diameters, respectively.

In vivo tumor growth was also evaluated in 3 mice/group by nuclear magnetic resonance (NMR) 24 hours after the second treatment. NMR imaging was performed with 1.5-T systems (Achieva, Philips) using similar pulse sequences. In all cases, coronal short $\tau$-inversion recovery sequences, axial turbo-spin-echo T2-weighted sequences and axial and coronal unenhanced turbo-spin-echo T1-weighted sequences were followed by axial and coronal contrast-enhanced turbo-spin-echo T1-weighted sequences (section thickness $2 \mathrm{~mm}$ ).

After 24 hours from the last treatment, tumors were excised and processed for IHC. Paraffin-embedded biopsies from NAMALWA and SU-DHL-4 xenografts were evaluated for HSP105 and Ki-67 expression as described in the previous paragraph. CD45, CD56, and Granzyme B immunostainings were performed on tissue sections from tumor xenografts using the following primary Abs: mouse anti-human CD45 (clones RP2/18 and RP2/22, Novocastra), rat anti-mouse CD56 (clone H28.123, Abcam), and rat anti-mouse Granzyme B (clone 16g6, eBioscience). Binding of primary Abs was revealed using the streptavidin-biotin-peroxidase complex method and 3-3'-diaminobenzidine as chromogenic substrate.

Experimental protocols were approved by the Ethical Committee for Animal Experimentation of the Fondazione IRCCS Istituto Nazionale dei Tumori di Milano, according to the Italian legislation (Legislative Order No. 116 of 1992, as amended), which implements the EU 86/109 Directive.

\section{Statistical analyses}

The 2-sided Student $t$ test $(P \leq .05)$ for unpaired data was used to detect statistically significant differences. The nonparametric Spearman rank correlation was used to measure the degree of association between
2 variables. Tumor volume data from the in vivo study were analyzed by 2-way ANOVA, and individual group comparisons were evaluated with the Bonferroni posttest. Statistical analyses were performed on Prism Version 5.0a software (GraphPad Software) for Macintosh Pro personal computer.

\section{Results}

\section{Induction of NHL cross-reacting Ab after DC-based vaccination}

We set out to determine whether vaccine-induced antitumor $\mathrm{Ab}$ response is directed against shared lymphoma-restricted antigens. Total Igs isolated from patients' serum samples, collected before and after vaccination, were biotinylated and then tested by IHC on allogeneic tumor specimens. IHC revealed tumor-restricted crossreactivity only when postvaccination Igs from responders were used (Figure 1Ai; supplemental Figure 1, available on the Blood Web site; see the Supplemental Materials link at the top of the online article). There were no differences in the immunostaining of pre- and postvaccination Igs from nonresponders (Figure 1Aii for a representative example). To determine whether the Ab-recognized tumor-specific antigens were expressed on the cell surface, biotinconjugated patients' Igs were tested by flow cytometry on live NHL, either cell lines or primary tumors, and normal B and T cells (Figure 1B). Vaccine-induced Ab responses to cell-surface tumorspecific antigens, evaluated as the ratio between post- and prevaccination Ig reactivity, were significantly higher in responders compared with nonresponders (Figure 1B). As shown in Figure 1B, the ability of postvaccination Igs from responders to recognize live tumor cell lines and primary cells increased by an average of 18-fold and 8-fold, respectively, compared with normal B cells (top). By contrast, post-vaccination Igs from nonresponders did not react with significantly enhanced efficiency on live tumor versus normal B cells (Figure 1B bottom). Western blot (WB) analyses of DOHH-2 cell extract using Igs from responders and nonresponders revealed a sharp band migrating at approximately $100-110 \mathrm{kDa}$ only when post-vaccination Igs from responders were used (Figure 1C). No reactivity was observed in the same molecular weight interval when prevaccination samples from these responders (Figure 1C left lanes 1) or pre- and postvaccine Igs from nonresponders (Figure 1C right) were used. Further proof of the induction of an antitumor $\mathrm{Ab}$ response in these patients was provided by the finding that postvaccination serum from responders mostly displayed a higher pattern of reactivity on the whole DOHH-2 proteome than the matched prevaccine samples (Figure $1 \mathrm{C}$ left).

\section{Serological identification of HSP105 in NHL}

For the discovery of proteins contained in the band migrating at approximately $110 \mathrm{kDa}$, which was differentially recognized by post-vaccination Igs from responders (Figure 1C left lanes 2), we applied an appropriately modified SERPA. DOHH-2 protein extract was separated by isoelectrofocusing into 24 fractions (F1-24, $\mathrm{pH} 3-9.57$ ) to be individually loaded on 1-dimensional SDS-PAGE and screened by WB analysis with responder's pre- and postvaccination sera that showed the highest differential reactivity against total DOHH-2 cell lysate (Figure 1C responder 14). An increased ability of post- versus prevaccination Igs to immunostain proteins migrating at approximately 100 to $110 \mathrm{kDa}$ was found in F6 (Figure 2Ai vs Figure 2Aii). MALDI-TOF-MS analysis, performed in that molecular weight range of a silver-stained preparative gel, identified in F6, and not in the nonreactive adjacent 
A
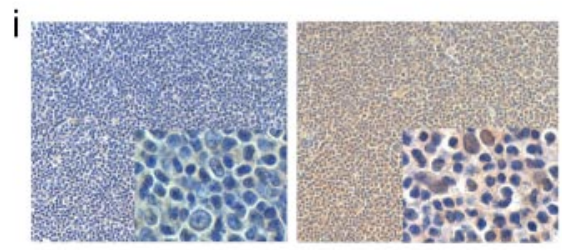

B
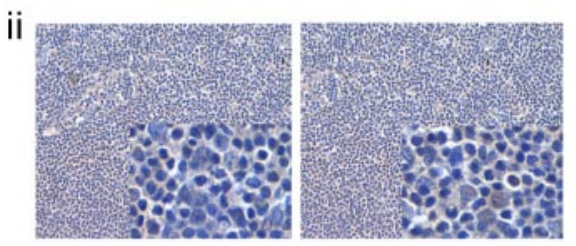

Figure 1. Antitumor cross-reactivity of vaccine-induced Igs in responders. (A) Representative IHC evaluations of biotin-conjugated pre(left) and postvaccination (right) Igs from responder 14 (i) and nonresponder 2 (ii) tested on allogeneic human FL specimens. Digital images were acquired with Nikon DN100 digital net camera and the Nikon Eclipse E400 microscope, and processed using Photoshop CS4 software version for Macintosh Pro personal computer. Original magnifications: panels, $\times 10$; insets, $\times 40$. (B) Flow cytometry of normal B (CD19) and T (CD3) cells, NHL cell lines (RL-19 and DOHH-2), and 4 different primary FLs (TM 1-4) using biotin-conjugated Igs from responders (top) and nonresponders (bottom) and FITC-labeled streptavidin as revealing system. Results are represented as the ratio between MFI of post- and prevaccination samples. Data are mean \pm SE of 4 independent experiments. (C) WB analyses of $\mathrm{DOHH}-2$ whole lysate probed with biotin-conjugated Igs purified from pre- (lanes 1 ) and post- (lanes 2 ) vaccination serum from responders $13,14,1$, and 5 (left) and nonresponders 2, 8, and 17 (right). Arrow indicates the differentially revealed proteins. CR indicates complete responder; and PR, partial responder.

C
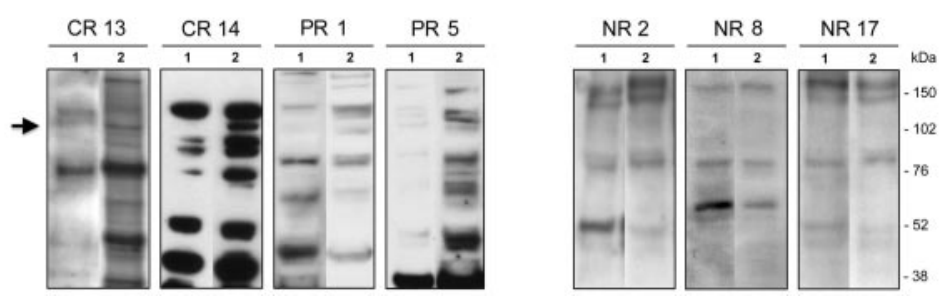

F5 and F7, U5S1 (component of the U5 small nuclear ribonucleoprotein particle), puromycin-sensitive aminopeptidase, minichromosome maintenance proteins-4 (a DNA helicase activity factor), all constitutively expressed in the nucleus or cytoplasm, and HSP105, as the potential proteins differentially recognized by post-vaccination Igs (Figure 2Bi). In light of the possibility that
HSP105 could be expressed on cell surface ${ }^{21}$ and the widely described roles of HSP family members in a broad range of neoplastic processes, ${ }^{10,13,22}$ we focused our study on understanding HSP105's involvement in B-NHL. The MS results were first validated by WB analysis of F6 with anti-HSP105 Ab (Figure 2Bii). In addition, immunoprecipitation of HSP105 from DOHH-2

A
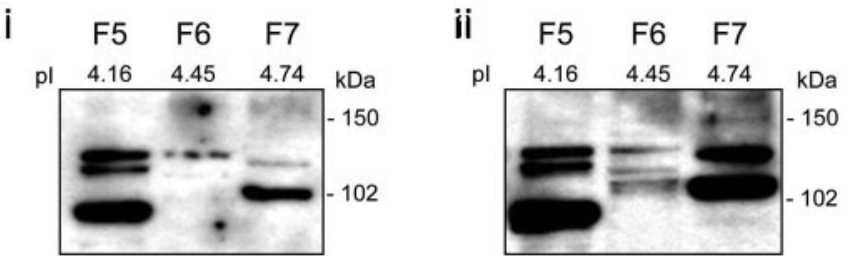

B
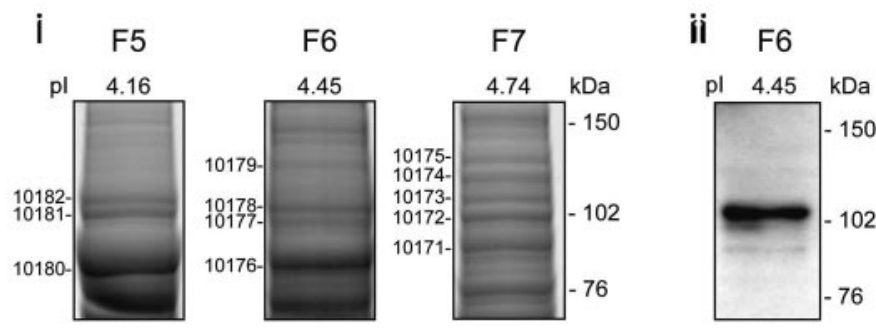

C

Figure 2. Serological identification of HSP105 in B-NHL. (A) WB analysis of OFFGEL fractions from DOHH-2 proteins (F5, F6, and F7) probed with prevaccination (i) and postvaccination (ii) Igs from responder 14. (B) Silver staining of F5, F6, and F7 preparative SDS-PAGE for MS of the numbered protein bands (i) and WB analysis of F6 probed with polyclonal anti-HSP105 Ab (ii). (C) Immunoprecipitation (IP) of HSP105 from $\mathrm{DOHH}-2$ whole cell lysate with polyclonal anti-HSP105 Ab, followed by WB analysis using pre- and postvaccination (Vax) Igs from responder 14 , or mouse anti-HSP105 mAb as control. pl indicates isoelectric point.

\section{IP: HSP105}
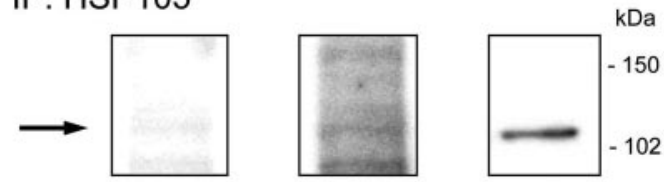

WB: Pre-Vax 
Figure 3. Flow cytometry of HSP105 in malignant lymphoid cell lines and normal cells. Surface (A) and intracellular (B) flow cytometric analyses of normal B cells, (1) low-grade B-cell NHL cell lines DOHH-2, (2) SC-1, (3) SU-DHL-6, and (4) KARPAS-422, (5) high-grade B-cell NHL cell lines RL-19, (6) SU-DHL-4, (7) RAJI, (8) NAMALWA, and (9) GRANTA-519, (10) Hodgkin lymphoma cell lines L-540 (11) and HDMYZ, (12) multiple myeloma cell line KMS-11, (13) normal T cells, (14) malignant T cell lines MOLT-4 (15), and SU-DHL-1 (16) stained with rabbit polyclonal antihuman HSP105 $\mathrm{Ab}$ and FITC-labeled antirabbit IgG. HSP105 relative MFI, calculated as the ratio between stained samples and negative control MFI values (left panels), and percentage of $\mathrm{HSP} 105^{+}$cells (right panels). Data are mean \pm SE of 6 independent experiments.

A
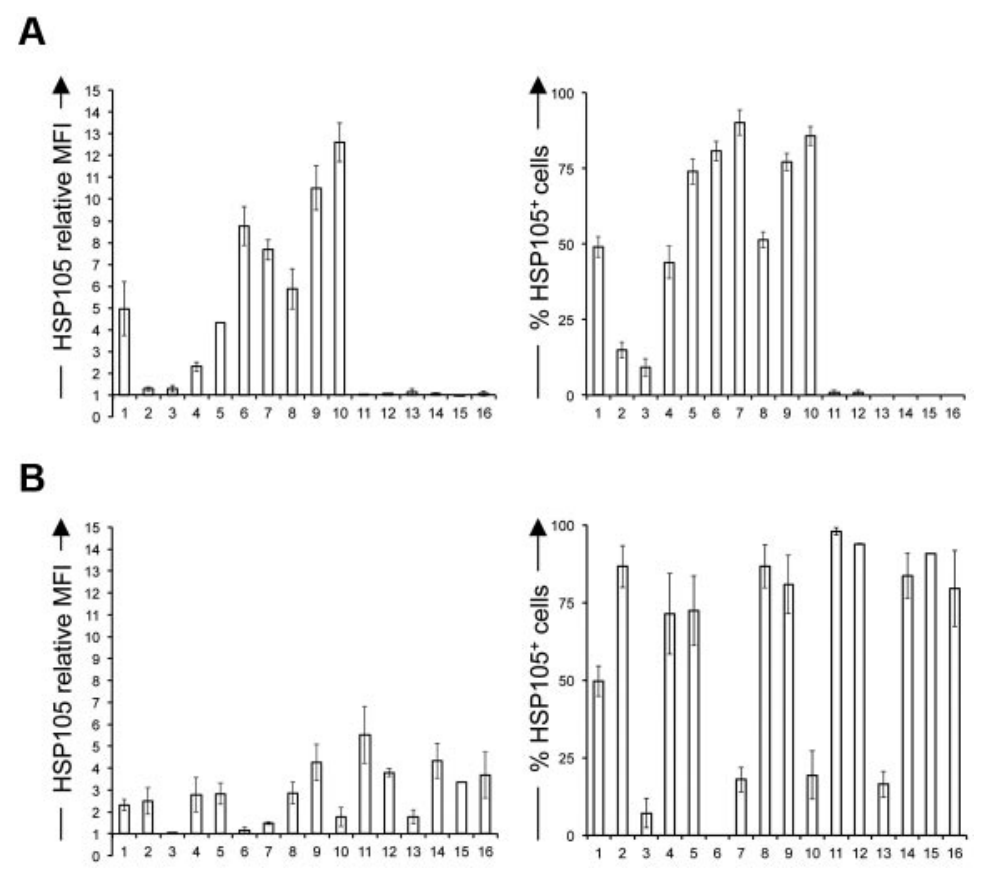

cell extract using a specific $\mathrm{Ab}$, followed by WB analysis with preand post-vaccination Igs from responder 14, confirmed that vaccination induced Abs directed against HSP105 (Figure 2C). The other differentially recognized proteins were not considered because of their intracellular expression and their already known structural functions. ${ }^{23-25}$

\section{Expression of HSP105 in human NHL cell lines and primary tumors}

To investigate the involvement of HSP105 in B-NHL, we studied its expression in a panel of human B-cell lymphoma cell lines (Figure 3 columns 2-13), including low- (Figure 3 columns 2-5) and high-grade NHLs (Figure 3, columns 6-10) and T-cell malignancies (Figure 3 columns 15 and 16) and their normal cell counterparts (Figure 3 columns 1 and 14) by flow cytometry. Interestingly, in B-NHL cell lines, the surface expression of HSP105, measured both as median fluorescence intensity (MFI; Figure 3A left) and positive cell percentage (Figure $3 \mathrm{~A}$ right), tended to increase in function of the degree of lymphoma aggressiveness. HSP105 was expressed on the surface of $\sim 50 \%$ of normal circulating B cells (Figure 3A right column 1). Donor PB T lymphocytes and cell lines derived from T-cell leukemia and lymphoma did not express HSP105 on their surface (Figure 3A columns 14-16). As expected, HSP105 was expressed within the cytoplasm of all the cell lines tested (Figure 3B). However, intracellular staining was heterogeneous in B-cell malignancies (Figure 3B columns 2-13), whereas it was comparably high in normal and neoplastic T cells (Figure 3B columns 14-16). WB analysis of these cell lines disclosed variable expression levels of both the $\alpha$ - and the $\beta$-isoforms of HSP105 (supplemental Figure 2 lanes 2-13, 15, and 16), whereas normal B and $\mathrm{T}$ cells preferentially expressed the constitutive cytoplasmic HSP105- $\alpha$ variant, represented by the one migrating at the highest molecular weight ${ }^{26,27}$ (supplemental Figure 2 lanes 1 and 14).

IHC was then used to compare the expression of HSP105 in 97 human primary B-NHL specimens (54 indolent and 43 aggressive lymphomas) and 26 nonmalignant lymph nodes. To quantify HSP105 reactivity in tissue sections, we defined a CS, ranging from 0 to 7, that took into account both HSP105-positive cell percentage and staining intensity. A CS $=4$ was arbitrarily considered as a threshold value that mainly discriminated between the low- and the high-grade B-NHLs (Figure 4A). IHC analysis of the tumor biopsies from vaccinated patients (10 of 18) revealed no significant differences in HSP105 expression level in function of their clinical outcome $(P=.625)$, indicating that the lack of response was not the result of a reduced lymphoma expression of HSP105 (Figure 4Ai, white represents responders; and gray, nonresponders). HSP105 expression was significantly higher in the aggressive compared with the indolent B-NHLs or normal lymph nodes (Figure 4Ai, $P<.001$, and supplemental Figure 3 for representative examples) and was directly correlated with the lymphoma histologic grade (Figure 4Ai, $P<.0001$, Spearman $\mathrm{r}=0.6951$ ). In addition, highproliferating tumors, characterized by an intense and widespread $\mathrm{Ki}-67^{+}$staining $\left(>50 \% \mathrm{Ki}-67^{+}\right.$nuclei), expressed HSP105 at significantly higher levels compared with the low-proliferating forms $\left(<50 \% \mathrm{Ki}-67^{+}\right.$nuclei; Figure 4Aii, $\left.P<.001\right)$. The lymphoma proliferation rate thus correlated with HSP105 expression levels (Figure 4Aii, $P<.0001$, Spearman $\mathrm{r}=0.7473$ ).

Even in indolent B-NHLs, where the structure of the follicles was preserved, HSP105 was preferentially expressed in the germinal center (GC) lymphocyte-enriched areas (Figure 4Bi right), which also stained strongly for Ki-67 (Figure 4Bi middle). Neoplastic follicles of low-grade FLs highlighted an intense expression of HSP105 in large cells corresponding to centroblasts, whereas small neoplastic centrocytes displayed a milder immunoreactivity (supplemental Figure 3A). Consistent with HSP105 expression paralleling the degree of B-cell clone aggressiveness, immunostaining revealed diffuse and intense HSP105 expression in the neoplastic infiltrates of DLBCL (supplemental Figure 3B) and BL (supplemental Figure 3C).

To corroborate the significant direct correlation between tumor aggressiveness and HSP105 expression levels, we used the histologic samples from a patient who progressed from a low-grade lymphoplasmacytic to a high-grade DLBCL after vaccination (patient 15). ${ }^{9}$ Interestingly, a substantial increase of expression and cell-surface localization of HSP105 was observed in the advanced (Figure 4Bii bottom right) compared with the diagnostic lymphoma 
A
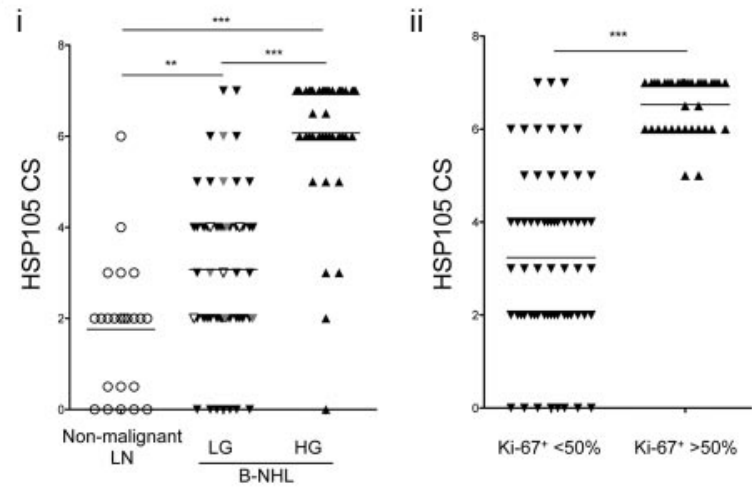

B

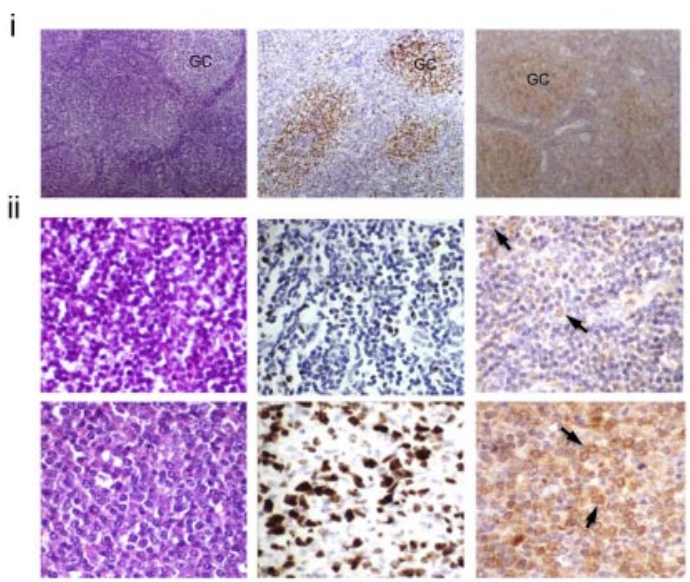

Figure 4. Immunohistochemistry of HSP105 expression in normal and neoplastic lymph nodes. (A) HSP105 expression levels measured by IHC using the defined CS in B-NHLs (97 cases) and nonmalignant lymph nodes (26 cases) plotted against tumor aggressiveness based on lymphoma histologic grade (i) or Ki-67 expression levels (ii). HSP105 CS in tumor biopsies from vaccinated patients is highlighted (white represents responders; and gray, nonresponders). The lines indicate the median values. Significant differences of HSP105 expression between groups were calculated with the unpaired 2-tailed Student $t$ test: ${ }^{\star \star} P \leq .01 ;{ }^{* \star \star} P \leq .001$. Correlation between HSP105 expression and lymphoma aggressiveness was determined with the Spearman rank correlation test. LN indicates lymph nodes; HG, high grade; and LG, low grade. (B) Representative IHC analyses of low- and high-grade B-NHLs, using streptavidin-biotin-HRP complex method with $3-3^{\prime}$ diaminobenzidine as a chromogen (brown signal). Hematoxylin and eosin (left), Ki-67 (middle), and HSP105 (right) staining in one case of FL (i) and lymphoplasmocytic lymphoma (ii) before (top) and after progression (bottom) toward a DLBCL. Arrows indicate HSP105 expression on lymphoma cell surface. Digital images were acquired with Nikon DN100 digital net camera and the Nikon Eclipse E400 microscope, and processed using Photoshop CS4 software version for Macintosh Pro personal computer. Original magnifications: $\mathrm{Bi}, \times 10$; and $\mathrm{Bii}, \times 20$.

(Figure 4Bii top right). The IHC results as a whole were in complete agreement with the quantitative flow cytometry analyses of cell-surface HSP105 levels on B-NHL cell lines (Figure 3A).

\section{Antitumor activity of HSP105 targeting}

The antitumor effects exerted by anti-HSP105 Ab were first investigated in vitro on DOHH-2, SU-DHL-4, and NAMALWA cell lines. Unexpectedly, the treatment did not provide any direct cytotoxic or cytostatic antitumor activity (Figure 5A-C). As shown in Figure 5A, no decrease in tumor cell proliferation rate was observed in both indolent and aggressive NHL cell lines treated with different concentrations of anti-HSP105 Ab. In keeping with this finding, neither tumor cell death nor cell cycle arrest was enhanced by the direct engagement of lymphoma cell-surface HSP105 (Figure 5B-C).
Furthermore, WB analyses of molecules mediating DNAdamage-repair functions and/or apoptosis (ie, RAD51, PARP, p53) did not reveal any significant modulation when SU-DHL-4 or NAMALWA cells were incubated in vitro with anti-HSP105 Ab at different time points (supplemental Figure 4).

However, because of the high surface expression of HSP105 in aggressive B-NHLs, we evaluated the immune-mediated antitumor effects of its targeting through a specific Ab in SCID mice subcutaneously implanted with SU-DHL-4 (Figure 5Di) or NAMALWA (Figure 5Dii) cells. Administration of anti-HSP105 Ab when xenografts became palpable significantly reduced the tumor burden in both xenografted models with respect to the control groups injected with the matched isotype Igs or saline (Figures 5D). After only 2 treatments with anti-HSP105 Ab, SU-DHL-4 and NAMALWA tumors displayed an average tumor shrinkage of $60 \%$ and $70 \%$, respectively, compared with the rabbit IgG-injected controls (Figure 5D; $P<.001$ ). These findings were also confirmed by NMR in both xenograft models (supplemental Figure 5). The greater NAMALWA tumor shrinkage compared with that of SU-DHL-4 (Figure 5D) was probably the result of the higher cell-surface expression level of HSP105 in NAMALWA cells (Figure 3A).

NAMALWA xenografts (supplemental Figure 6A), as defined by CD45 staining (supplemental Figure 6B), expressed Ki-67 (supplemental Figure 6C) and HSP105 (supplemental Figure 6D) at levels superimposable on those observed in human high-grade primary B-NHL specimens (Figure 4Bii bottom; supplemental Figure 3C). A marked increase of necrotic areas was detected in anti-HSP105 Ab-treated xenografts with respect to the controls, as revealed by hematoxylin and eosin stainings (Figure 6Ai bottom vs top, for representative examples). Under low power, untreated tumors displayed the typical "starry-sky" pattern of BL, indicating the presence of apoptotic tumor cells (Figure 6Ai top). On HSP105 targeting, the tumor xenografts showed signs of single-cell necrosis and extended necrotic foci, which involved both lymphoid neoplastic cells and the branching fibrovascular stromal network (Figure 6Ai bottom). Necrotic changes characterizing anti-HSP105 Abtreated lymphomas were associated with a significant infiltration of NK cells, highlighted by Granzyme B (Figure 6Aii bottom vs top) and CD56 immunostaining (Figure 6Aiii bottom vs top), suggesting the contribution of Ab-dependent cell-mediated cytotoxicity (ADCC) to the antitumor activity of this Ab-based immunotherapy, as already described for other Abs in different tumor models. ${ }^{28}$ Quantitative evaluation of tumor-infiltrating Granzyme $\mathrm{B}^{+}$(Figure 6Bi) and $\mathrm{CD}^{+} 6^{+}$cells (Figure 6Bii), which were counted in 5 high-power microscopic fields in sections from anti-HSP105 Aband isotype Ig-treated xenografts, reproducibly confirmed this finding.

\section{Discussion}

In the present study, we identify HSP105 as a novel potential therapeutic biotarget in B-NHLs. Specifically, starting from the observation that DC-based vaccination in NHL patients enhanced the frequency of circulating Abs directed against shared lymphoma antigens only in responders, an appropriately modified SERPA applied to a NHL cell line proteome revealed HSP105 as one of the possible responders' Ab-targeted proteins. The cell-surface expression of HSP105 correlated with B-NHL proliferation rate and aggressiveness in both cell lines and primary tumors. Despite the lack of direct proapoptotic and cytostatic in vitro activity of an 
Figure 5. In vitro and in vivo antilymphoma activity of anti-HSP105 Ab. (A) DOHH-2 (i), SU-DHL-4 (ii), and concentrations of the anti-HSP105 Ab formulation for in vivo use; and after being stained with $\mathrm{PI}$, viable cells in culture $\left(\mathrm{PI}^{-}\right)$were counted by flow cytometry using a calibrated suspension of fluorescent microspheres at the indicated time points ( $\mathrm{h}$ indicates hours). The plots show the means of 3 independent experiments. (B) Flow cytometric determinations of apoptosis of the cell cultures described in panel A costained with FITC-labeled annexin $\mathrm{V}$ (ANN) and $\mathrm{PI}$. Average percentages of $\mathrm{ANN}^{-} \mathrm{PI}^{-}$(white), $\mathrm{ANN}^{+} \mathrm{PI}^{-}$(light gray), $\mathrm{ANN}^{+} \mathrm{PI}^{+}$(dark gray), and $\mathrm{ANN}^{-} \mathrm{PI}^{+}$(black) cells in cultures, calculated from the results of 3 independent experiments, are shown. (C) Flow cytometry-based cell-cycle analyses of DOHH-2 (i), SU-DHL-4 (ii), and NAMALWA (iii) cells after 72-hour incubation with anti-HSP105 Ab at the indicated concentrations. Average frequencies of cells in $\mathrm{G}_{1}$ (black), $S$ (white), and $G_{2}$ (gray) cell-cycle phases and SD of 3 independent experiments are shown. (D) SU-DHL-4 (i) and NAMALWA (ii) growth in SCID mice treated with 3 intraperitoneal injections of $\mathrm{NaCl}$ solution (ctrl), $250 \mu \mathrm{g}$ anti-HSP105 $\mathrm{Ab}$, or matched isotype Igs (rabbit $\lg \mathrm{G}$ ) every 4 days (black arrows). Average tumor volumes (measured with calipers) and SE of 2 (SU-DHL-4 model) and 3 (NAMALWA model) independent experiments, in which 6 mice per group were analyzed. Statistically significant differences were calculated by using the 2-way ANOVA: ${ }^{* *} P<.01 ;{ }^{* * *} P<.001$. Black stars represent anti-HSP105 Ab versus ctrl. NAMALWA (iii) cells were treated with the indicated anti-HSP105 $A b$ versus rabbit IgG; and gray stars,

\section{A}
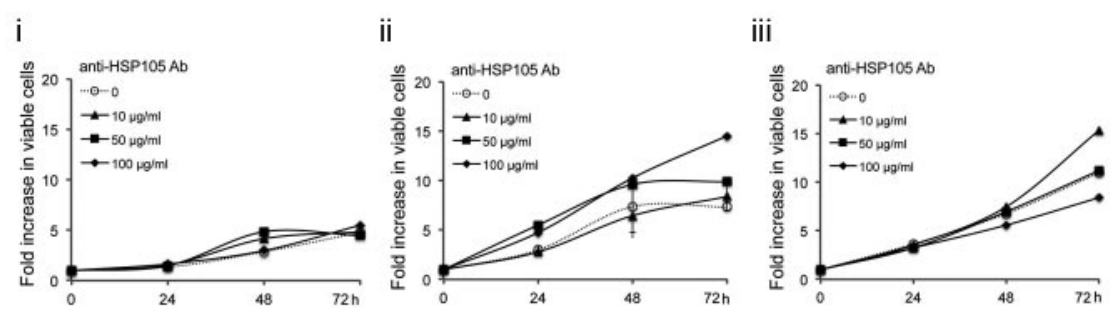

\section{B}
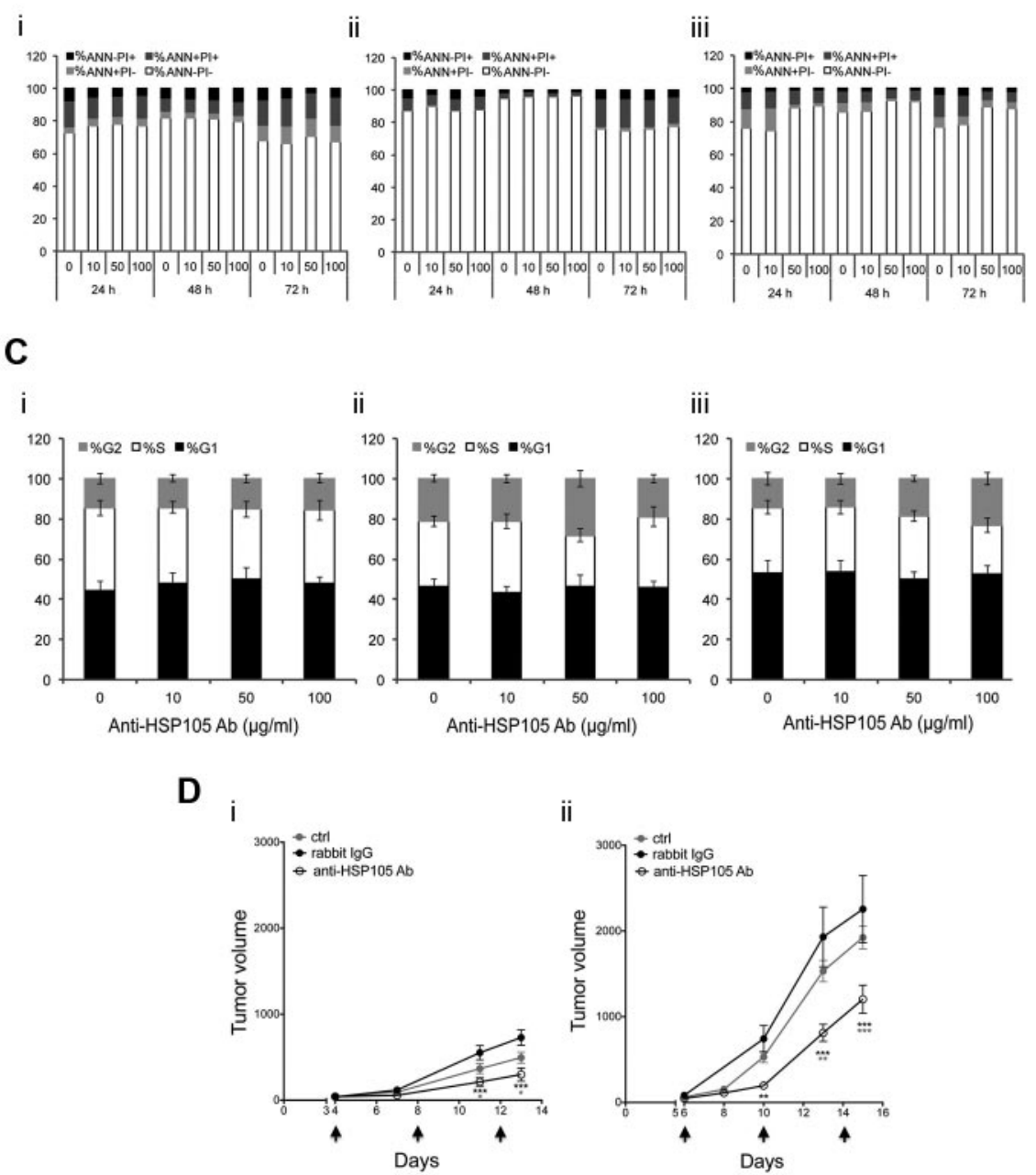

HSP105-specific Ab, its administration in SCID mice significantly delayed and impaired the growth of aggressive human B-NHL xenografts. Because HSP105 engagement by this Ab favored the recruitment of $\mathrm{CD}_{56}{ }^{+}$, Granzyme B-releasing killer cells at the tumor site, we speculate that ADCC may have exerted a major role in promoting the extended necrosis observed in anti-HSP105 Ab-treated tumor tissue compared with the control tumor biopsies.

The management of NHL patients has been considerably improved by the introduction of mAb therapy. Addition of the anti-CD20 mAb rituximab to conventional and high-dose chemotherapy protocols has significantly ameliorated the prognosis of B-NHLs. ${ }^{5}$ Despite this clinical success, resistance occurs in approximately half of the treated patients, resulting in nonresponse to treatment or early relapse. ${ }^{6,7}$ Hence, different NHL biotargets and more potent $\mathrm{mAbs}$ are continuously being sought.
The finding that DC vaccination induced the development of lymphoma-specific Abs only in responders led to the serological discovery of novel potential NHL therapeutic targets through exploitation of the $\mathrm{Ab}$ repertoires from vaccinated patients. For this purpose, we applied a high-resolution 2-step SERPA to circumvent the limitations of the classic 2-dimensional SDS-PAGE approach to barely detect low-molecular-weight proteins and those with extreme $\mathrm{pH}$, as well as low-abundance and poorly soluble hydrophobic proteins, such as cell-surface proteins..$^{29,30}$ This strategy successfully revealed a pattern of increased reactivity for post- compared with pre-vaccination Abs from responders on a B-NHL-proteome, and MS identified HSP105 as one of the cell-surface antigens differentially recognized. There is little information on how HSP regulation is subverted in cancer and how it affects the molecular events involved in tumor growth, invasiveness, and metastasis. ${ }^{11}$ 


\section{A}

i

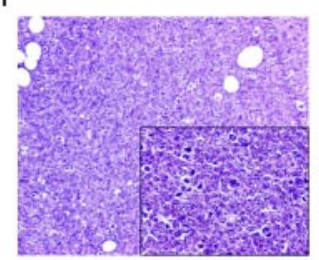

ii

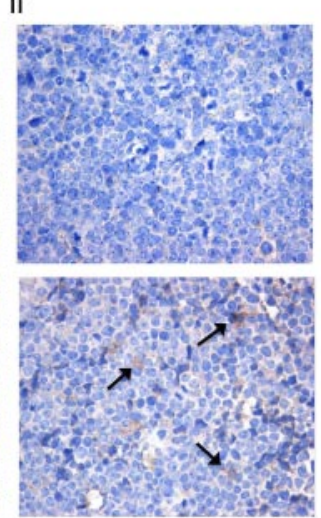

iii

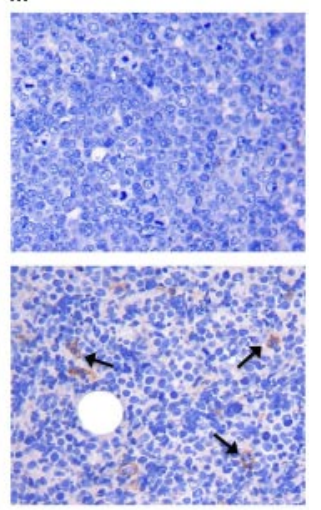

Figure 6. Antitumor effects of HSP105 targeting in the NAMALWA model. (A) IHC of NAMALWA xenografts from control rabbit IgG- (top) and anti-HSP105 Ab-treated (bottom) animals. Representative hematoxylin and eosin (i), Granzyme B (ii), and CD56 (iii) stainings. Digital images were acquired with Leica DFC320 digital camera and Leica DM2000 microscope, and processed using Photoshop CS4 software version for Macintosh Pro personal computer. Original magnifications: i, $\times 200$; ii,iii, $\times 400$; and insets, $\times 400$. (B) Absolute number of Granzyme B+ (i) and $\mathrm{CD}^{+} 6^{+}$(ii) cells in sections from control rabbit IgG- and anti-HSP105 Ab-treated xenografts. Immunoreactive cells (ie, yellow/brown-stained cells) were counted in 5 high-power microscopic fields (original magnification, $\times 400$ ) for each condition, and the result was expressed as an average. The boxes extend from the 25th to the 75th percentiles, the lines indicate the median values, and the whiskers indicate the range of values. Results from 2 independent experiments are shown. Statistically significant differences between groups were assessed with the unpaired 2-tailed Student $t$ test: ${ }^{* \star} P<.001$

\section{B}

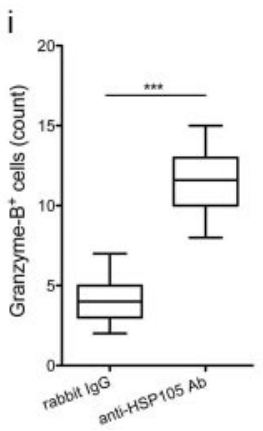

ii

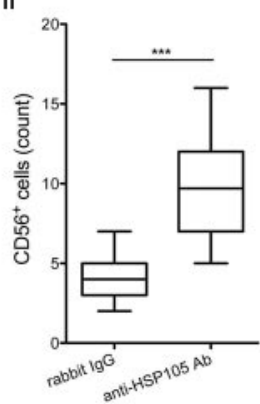

However, it seems that HSP can modulate both proapoptotic and antiapoptotic pathways. ${ }^{16,13}$ In addition, extracellular or membranebound HSPs, mainly HSP90 and HSP72, can induce T-cell activation because of their carrier function for antigenic peptides and their ability to deliver direct immune-activating signals. ${ }^{18,31,32}$ It is therefore possible that DC vaccination provided HSP105peptide complex to the host immune system and/or activated it toward multiple tumor-associated antigens, including HSP105, and favored the onset of a clinical response.

Human HSP105 is a high-molecular-weight chaperone protein expressed at constitutively low levels as a cytoplasmic $\alpha$-isoform and as an inducible nuclear $\beta$-isoform on exposure to various forms of stress. ${ }^{27}$ However, in several solid tumors, including melanoma, breast, thyroid, and gastroenteric cancers, HSP105 is constitutively overexpressed $^{33-35}$ and exerts antiapoptotic functions. ${ }^{36-38}$ HSP105 had also been previously defined by serological-recombinant expression cloning technique, using serum samples from pancreatic and colorectal carcinoma patients. ${ }^{33}$ This provides a further illustration of its immunogenic properties in cancer patients. Importantly, several preclinical studies have demonstrated that preventive immunization against HSP105 provides a significant antitumor activity. ${ }^{39,40}$ A very recent report has demonstrated the expression of HSP105 in the cytoplasm and within the nucleus of Hodgkin/Reed-Sternberg cells of classic Hodgkin lymphoma ${ }^{15}$; however, no previous studies have already shown the cell surface expression of HSP105 in B-NHLs.

Here, we demonstrated a significant direct correlation between HSP105 expression levels and B-NHL aggressiveness and proliferation. Its up-regulation during disease progression from indolent to aggressive NHL further corroborated these findings, which, collectively, point to HSP105 as a novel candidate biomarker of lymphoma aggressiveness. Our results in lymphoma are in line with previous demonstrations in solid tumors of the association between HSP105 up-regulation in tumor tissue and disease progression. ${ }^{35,41}$ The finding of an HSP105 intermediate cell-surface expression on donor circulating B cells, which are professional antigen-presenting cells, could be explained by the already reported function of HSPs in antigen cross-presentation..$^{32,42}$

In agreement with a recent report demonstrating the ability of HSP90 to stabilize BCL-6 in normal centroblasts during the affinity maturation process, ${ }^{43}$ the preferential localization of HSP105 within the GCs of low-grade lymphomas and normal lymph nodes (data not shown) indicates that GC reaction may be governed by a stress response that may itself be involved in the physiopathology of GC-derived B-cell lymphomas. In keeping with this hypothesis, overexpression of the alternative splicing variant HSP105 $\beta$ has been found to stimulate the phosphorylation of STAT3, ${ }^{26,27,44,45}$ which constitutes a crucial signaling node of B-NHLs. ${ }^{46-48}$ Increasing evidence is pointing to the stress response pathway as an additional hallmark of cancer and a promising new target for anticancer therapy. ${ }^{13}$ Tumor cells, indeed, require HSPs to maintain normal homeostasis of their altered proteome and cope with the potentially lethal conditions in which they are forced to grow, and for the correct maturation and function of several oncoproteins. ${ }^{13,49}$

Remarkably, direct targeting of HSP105 with a specific Ab demonstrated a significant antitumor activity against highly aggressive human lymphoma cell lines in vivo. No significant off-target toxicity was documented after targeting HSP105 in xenografted lymphoma mouse models. However, the mechanisms accounting for the in vivo antitumor effects of the anti-HSP105 Ab used in our study seem to essentially rely on its ability to activate ADCC at the tumor site. The engagement of surface-HSP105 with this Ab, 
indeed, did not alter any of the crucial signaling pathways associated with tumor-cell proliferation and survival as well as DNA-damage response, including those mediated by p53, PARP, RAD51, and MAPK and AKT (data not shown).

The development of novel functional anti-HSP105 mAbs as well as the use of specific blocking peptides will clarify the functional role of HSP105 in B-NHLs and also show whether it can be added to the growing list of not merely oncogenic molecules ${ }^{13,49}$ that may represent equally valid targets for anticancer therapy.

\section{Acknowledgments}

The authors thank the patients for their generous participation in this study and Piera Aiello for excellent technical assistance.

This work was supported in part by Associazione Italiana per la Ricerca sul Cancro, Milan, Italy, and Fondazione Michelangelo,
Milan, Italy (research funding, M.D.N.). R.Z. was supported by Associazione Marco Semenza, Milan, Italy (fellowship).

\section{Authorship}

Contribution: R.Z., S.M.P., A.M.G., and M.D.N. designed the research and wrote the paper; R.Z., G.C.G., L.C., M.T., A.D.C., C.T., A.M., C.C.-S., M.M., and I.B. performed experiments; and R.Z., S.M.P., and M.D.N. analyzed results and compiled the figures.

Conflict-of-interest disclosure: The authors declare no competing financial interests.

Correspondence: Massimo Di Nicola, C. Gandini Medical Oncology, Bone Marrow Transplantation Unit, Fondazione IRCCS Istituto Nazionale dei Tumori di Milano, Via Venezian, 1, 20133 Milan, Italy; e-mail: massimo.dinicola@istitutotumori.mi.it.

\section{References}

1. Shaffer AL, Rosenwald A, Staudt LM. Lymphoid malignancies: the dark side of B-cell differentiation. Nat Rev Immunol. 2002;2(12):920-932.

2. Küppers R. Mechanisms of B-cell lymphoma pathogenesis. Nat Rev Cancer. 2005;5:251-262.

3. Multani P, White CA, Grillo-Lopez A. Non-Hodgkin's lymphoma: review of conventional treatments. Curr Pharm Biotechnol. 2001;2(4):279291.

4. Leget GA, Czuczman MS. Use of rituximab, the new FDA-approved antibody. Curr Opin Oncol. 1998;10(6):548-551.

5. Cheson BD, Leonard JP. Monoclonal antibody therapy for B-cell non-Hodgkin's lymphoma. N Engl J Med. 2008;359(6):613-626.

6. McLaughlin P, Grillo-Lopez AJ, Link BK, et al. Rituximab chimeric anti-CD20 monoclonal antibody therapy for relapsed indolent lymphoma: half of patients respond to a four-dose treatment program. J Clin Oncol. 1998;16(8):2825-2833.

7. Davis TA, Grillo-Lopez AJ, White CA, et al. Rituximab anti-CD20 monoclonal antibody therapy in non-Hodgkin's lymphoma: safety and efficacy of re-treatment. J Clin Oncol. 2000;18(17):31353143.

8. Tarella C, Passera R, Magni M, et al. Risk factors for the development of secondary malignancy after high-dose chemotherapy and autograft, with or without rituximab: a 20-year retrospective follow-up study in patients with lymphoma. J Clin Oncol. 2011;29(7):814-824.

9. Di Nicola M, Zappasodi R, Carlo-Stella C, et al. Vaccination with autologous tumor-loaded dendritic cells induces clinical and immunologic responses in indolent B-cell lymphoma patients with relapsed and measurable disease: a pilot study. Blood. 2009;113(1):18-27.

10. Whitesell L, Lindquist SL. HSP90 and the chaperoning of cancer. Nat Rev Cancer. 2005;5(10): 761-772.

11. Ciocca DR, Calderwood SK. Heat shock proteins in cancer: diagnostic, prognostic, predictive, and treatment implications. Cell Stress Chaperones. 2005;10(2):86-103.

12. Bohonowych JE, Gopal U, Isaacs JS. Hsp90 as a gatekeeper of tumor angiogenesis: clinical promise and potential pitfalls. J Oncol. 2010;2010: 412985.

13. Luo J, Solimini NL, Elledge SJ. Principles of cancer therapy: oncogene and non-oncogene addiction. Cell. 2009;136(5):823-837.

14. Trepel J, Mollapour M, Giaccone G, Neckers L. Targeting the dynamic HSP90 complex in cancer. Nat Rev Cancer. 2010;10(8):537-549.

15. Santon A, Garcia-Cosio M, Cristobal E, Pascual
A, Muriel A, Garcia-Larana J. Expression of heat shock proteins in classical Hodgkin lymphoma: correlation with apoptotic pathways and prognostic significance. Histopathology. 2011;58(7):10721080.

16. Khalil AA, Kabapy NF, Deraz SF, Smith C. Heat shock proteins in oncology: diagnostic biomarkers or therapeutic targets? Biochim Biophys Acta. 2011;1816(2):89-104

17. Cheson BD, Pfistner B, Juweid ME, et al. Revised response criteria for malignant lymphoma. J Clin Oncol. 2007;25(5):579-586.

18. Zappasodi R, Pupa SM, Ghedini GC, et al. Improved clinical outcome in indolent B-cell lymphoma patients vaccinated with autologous tumor cells experiencing immunogenic death. Cancer Res. 2010;70:9062-9072.

19. Ros A, Faupel M, Mees H, et al. Protein purification by off-gel electrophoresis. Proteomics. 2002; 2(2):151-156.

20. Caccia D, Micciche F, Cassinelli G, Mondellini P, Casalini P, Bongarzone I. Dasatinib reduces FAK phosphorylation increasing the effects of RPI-1 inhibition in a RET/PTC1-expressing cell line. $\mathrm{Mo}$ Cancer. 2010;9:278.

21. Weidle UH, Maisel D, Klostermann S, Schiller C, Weiss EH. Intracellular proteins displayed on the surface of tumor cells as targets for therapeutic intervention with antibody-related agents. Cancer Genomics Proteomics. 2011;8(2):49-63.

22. Cappello F, David S, Peri G, et al. Hsp60: molecular anatomy and role in colorectal cancer diagnosis and treatment. Front Biosci. 2011;3:341351.

23. Musahl C, Schulte D, Burkhart R, Knippers R. A human homologue of the yeast replication protein Cdc21: interactions with other Mcm proteins. Eur J Biochem. 1995;230(3):1096-1101.

24. Achsel T, Ahrens K, Brahms H, Teigelkamp S, Luhrmann R. The human U5-220kD protein (hPrp8) forms a stable RNA-free complex with several U5-specific proteins, including an RNA unwindase, a homologue of ribosomal elongation factor EF-2, and a novel WD-40 protein. Mol Cell Biol. 1998;18(11):6756-6766.

25. Thompson MW, Tobler A, Fontana A, Hersh LB Cloning and analysis of the gene for the human puromycin-sensitive aminopeptidase. Biochem Biophys Res Commun. 1999;258(2):234-240.

26. Saito Y, Yamagishi N, Hatayama T. Different localization of Hsp105 family proteins in mammalian cells. Exp Cell Res. 2007;313(17):37073717.

27. Saito Y, Yamagishi N, Hatayama T. Nuclear localization mechanism of Hsp105beta and its pos- sible function in mammalian cells. J Biochem. 2009;145(2):185-191.

28. Nagayama S, Fukukawa C, Katagiri T, et al. Therapeutic potential of antibodies against FZD 10, a cell-surface protein, for synovial sarcomas. Oncogene. 2005;24(41):6201-6212.

29. Klade CS. Proteomics approaches towards antigen discovery and vaccine development. Curr Opin Mol Ther. 2002;4(3):216-223.

30. Hamrita B, Chahed K, Kabbage M, et al. Identification of tumor antigens that elicit a humoral immune response in breast cancer patients' sera by serological proteome analysis (SERPA). Clin Chim Acta. 2008;393(2):95-102.

31. Suto R, Srivastava PK. A mechanism for the specific immunogenicity of heat shock protein-chaperoned peptides. Science. 1995;269(5230):15851588.

32. Schmitt E, Gehrmann M, Brunet M, Multhoff G, Garrido $C$. Intracellular and extracellular functions of heat shock proteins: repercussions in cancer therapy. J Leukoc Biol. 2007;81(1):15-27.

33. Nakatsura T, Senju S, Yamada K, Jotsuka T, Ogawa M, Nishimura Y. Gene cloning of immunogenic antigens overexpressed in pancreatic cancer. Biochem Biophys Res Commun. 2001; 281(4):936-944.

34. Kai M, Nakatsura T, Egami H, Senju S, Nishimura Y, Ogawa M. Heat shock protein 105 is overexpressed in a variety of human tumors. Oncol Rep. 2003;10(6):1777-1782.

35. Park H-S, Park C-H, Choi B-R, et al. Expression of heat shock protein 105 and 70 in malignant melanoma and benign melanocytic nevi. J Cutan Pathol. 2009;36(5):511-516.

36. Hosaka S, Nakatsura T, Tsukamoto H, Hatayama T, Baba H, Nishimura Y. Synthetic small interfering RNA targeting heat shock protein 105 induces apoptosis of various cancer cells both in vitro and in vivo. Cancer Sci. 2006;97(7):623-632.

37. Yamagishi $\mathrm{N}$, Ishihara $\mathrm{K}$, Saito $\mathrm{Y}$, Hatayama T. Hsp105 family proteins suppress staurosporineinduced apoptosis by inhibiting the translocation of Bax to mitochondria in HeLa cells. Exp Cell Res. 2006;312(17):3215-3223.

38. Yamagishi N, Goto K, Nakagawa S, Saito Y, Hatayama T. Hsp105 reduces the protein aggregation and cytotoxicity by expanded-polyglutamine proteins through the induction of Hsp70. Exp Cell Res. 2010;316:2424-2433.

39. Wang X-Y, Chen X, Manjili MH, Repasky E, Henderson R, Subjeck JR. Targeted immunotherapy using reconstituted chaperone complexes of heat shock protein 110 and melanomaassociated antigen gp100. Cancer Res. 2003; 63(10):2553-2560. 
40. Miyazaki M, Nakatsura T, Yokomine K, et al. DNA vaccination of HSP105 leads to tumor rejection of colorectal cancer and melanoma in mice through activation of both CD4 T cells and CD8 T cells. Cancer Sci. 2005;96(10):695-705.

41. Slaby O, Sobkova K, Svoboda M, et al. Significant overexpression of Hsp110 gene during colorectal cancer progression. Oncol Rep. 2009; 21(5):1235-1241.

42. Li Z, Menoret A, Srivastava P. Roles of heatshock proteins in antigen presentation and crosspresentation. Curr Opin Immunol. 2002;14(1):4551.

43. Cerchietti LC, Lopes EC, Yang SN, et al. A purine scaffold Hsp90 inhibitor destabilizes BCL-6 and has specific antitumor activity in BCL-6-dependent B cell lymphomas. Nat Med. 2009;15(12): 1369-1376.

44. Yamagishi N. Hsp105 but not Hsp70 family proteins suppress the aggregation of heat-denatured protein in the presence of ADP. FEBS Lett. 2003; 555(2):390-396.

45. Yamagishi N, Fujii H, Saito Y, Hatayama T. Hsp105beta upregulates hsp70 gene expression through signal transducer and activator of transcription-3. FEBS J. 2009;276(20):5870-5880.

46. Alas $\mathrm{S}$, Bonavida B. Rituximab inactivates signal transducer and activation of transcription 3 (STAT3) activity in B-non-Hodgkin's lymphoma through inhibition of the interleukin 10 autocrine/ paracrine loop and results in down-regulation of $\mathrm{Bcl}-2$ and sensitization to cytotoxic. Cancer Res. 2001;61(13):5137-5144.

47. Vanasse GJ, Winn RK, Rodov S, et al. Bcl-2 overexpression leads to increases in suppressor of cytokine signaling- 3 expression in B cells and de novo follicular lymphoma. Mol Cancer Res. 2004; 2(11):620-631.

48. Scuto A, Kujawski M, Kowolik C, et al. STAT3 Inhibition is a therapeutic strategy for $\mathrm{ABC}$-like diffuse large B-cell lymphoma. Cancer Res. 2011; 71(9):3182-3188.

49. Hanahan D, Weinberg RA. Hallmarks of cancer: the next generation. Cell. 2011;144(5):646-674. 


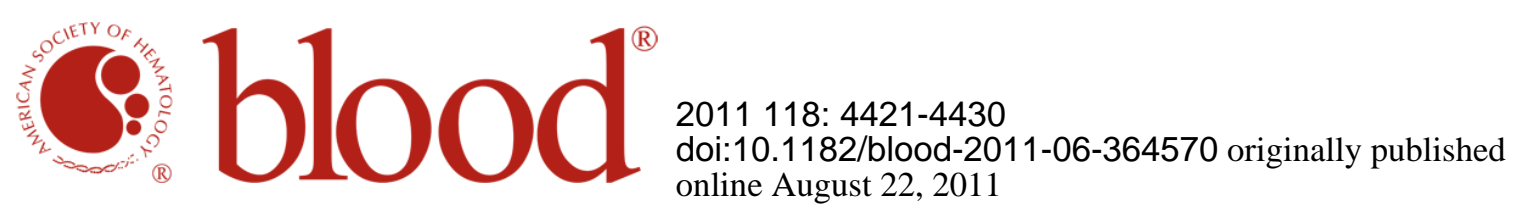

\section{Serological identification of HSP105 as a novel non-Hodgkin lymphoma therapeutic target}

Roberta Zappasodi, Italia Bongarzone, Gaia C. Ghedini, Lorenzo Castagnoli, Antonello D. Cabras, Antonella Messina, Monica Tortoreto, Claudio Tripodo, Michele Magni, Carmelo Carlo-Stella, Alessandro M. Gianni, Serenella M. Pupa and Massimo Di Nicola

Updated information and services can be found at:

http://www.bloodjournal.org/content/118/16/4421.full.html

Articles on similar topics can be found in the following Blood collections

Immunobiology (5424 articles)

Lymphoid Neoplasia (2404 articles)

Information about reproducing this article in parts or in its entirety may be found online at:

http://www.bloodjournal.org/site/misc/rights.xhtml\#repub_requests

Information about ordering reprints may be found online at:

http://www.bloodjournal.org/site/misc/rights.xhtml\#reprints

Information about subscriptions and ASH membership may be found online at:

http://www.bloodjournal.org/site/subscriptions/index.xhtml

Blood (print ISSN 0006-4971, online ISSN 1528-0020), is published weekly by the American Society of Hematology, 2021 L St, NW, Suite 900, Washington DC 20036.

Copyright 2011 by The American Society of Hematology; all rights reserved. 